\title{
EFFECT OF TRICARBOXYLIC ACID CYCLE INTERMEDIATES ON ACETIC ACID PRODUCTION BY ACETOBACTER ACETI
}

\author{
YONG SOO PARK AND KIYOSHI TODA* \\ Institute of Applied Microbiology, \\ University of Tokyo, 1-1-1 Yayoi, Bunkyo-ku, Tokyo 113, Japan
}

(Received January 23, 1990)

\begin{abstract}
The stimulative effect of tricarboxylic acid (TCA) cycle intermediates on the acetic acid production of Acetobacter aceti M23 was investigated in batch and continuous cultures. Cell growth was hardly affected by the supplement of the TCA cycle intermediates; however, the acetic acid production rate in a continuous culture at the dilution rate of $0.097 \mathrm{~h}^{-1}$ increased 3.2 times in the medium supplemented with $2 \mathrm{~mm}$ citric acid. The optimum concentration of citric acid as an additive in the medium was $10 \mathrm{~mm}$. Acetic acid production was enhanced by adding citric acid only when there was an insufficient supply of oxygen.
\end{abstract}

It has been reported that some tricarboxylic acid (TCA) cycle intermediates added to a medium promotes acetic acid production(1). Samson et al.(7) also observed that the intermediates of TCA cycle such as succinate and citrate enhanced the phosphate uptake by a yeast strain.

In the present study we reassessed their effects in promoting the acetic acid production by Acetobacter aceti in batch and continuous cultures. Particularly we investigated the effects of some of the TCA cycle intermediates on the rates of cell growth and acetic acid production.

\section{MATERIALS AND METHODS}

Microorganism. Acetobacter aceti M23 was provided from Nakano Vinegar Co. Ltd. (Japan).

Culture medium. The composition of a basal medium was in gram per liter: glucose, 1; yeast extract, 5; peptone, 2; acetic acid, 10; ethanol, 47.4 ( $\mathrm{pH} \mathrm{3.3).} \mathrm{Acetic}$ acid and ethanol were added to the basal medium sterilized with steam for $15 \mathrm{~min}$

* Address reprint requests to: Dr. Kiyoshi Toda, Institute of Applied Microbiology, University of Tokyo, 1-1-1 Yayoi, Tokyo 113, Japan. 
at $121^{\circ} \mathrm{C}$. Citric, oxaloacetic, maleic, fumaric, $\alpha$-ketoglutaric, succinic and pyruvic acids as the intermediate substances in the TCA cycle were added to the respective basal media to a final concentration of $10 \mathrm{~mm}$ to investigate their effects on acetic acid production. Adding the acids changed the medium $\mathrm{pH}$ only negligibly.

Culture method. A loop of slant culture of A. aceti M23 was inoculated in a test tube containing $10 \mathrm{ml}$ of basal medium and incubated $48 \mathrm{~h}$ at $30^{\circ} \mathrm{C}$. The test tube culture was repeated twice with an inoculation ratio of $5 \%$. The cells at a logarithmic growth phase were used for measuring their responses to additives throughout the experiment. The test tube and shake flask cultures were carried out for $140 \mathrm{~h}$ and $130 \mathrm{~h}$, respectively.

Bubble column bioreactor. A bubble column reactor was used for a continuous culture of $A$. aceti. The column was $5.0 \mathrm{~cm}$ i.d. and $28.5 \mathrm{~cm}$ high. The working volume was $322 \mathrm{ml}$ and the liquid temperature was controlled at $30^{\circ} \mathrm{C}$. To prevent an evaporation loss of ethanol from the reactor the bubble column was equipped with a liquid condenser. The air sparging was carried out by a glass ball filter located at the bottom with an aeration rate of $1 \mathrm{vvm}$. The oxygen partial pressure of air was set at $0.21 \mathrm{~atm}$ or $0.8 \mathrm{~atm}$. The volumetric oxygen transfer coefficient was about $50 \mathrm{~h}^{-1}$ as determined by the sodium sulfite method. An oxygen concentrator (Hai Sanso TO-90, Teijin, Tokyo, Japan) was used to elevate the oxygen partial pressure.

Assay. Concentrations of acetic acid and ethanol were measured by the gas chromatographic method using iso-butanol as an internal standard substance. Acetic acid also measured titrimetrically with $0.1 \mathrm{~N} \mathrm{NaOH}$ using $0.2 \%$ phenol red solution as an indicator. The turbidity of the culture was measured as an optical density at $660 \mathrm{~nm}$, and converted to the dry cell concentration using the calibration curve between dry cell weight and optical density.

\section{RESULTS AND DISCUSSION}

Effect of TCA cycle intermediates on the specific growth rate and acetic acid production rate

The test tube culture of Acetobacter cells was conducted with the addition of the TCA cycle intermediates in the medium. The concentration of the additives was fixed at $10 \mathrm{~mm}$. The maximum specific growth rate $\left(\mu_{\mathrm{m}}\right)$, the concentrations of cells and acetic acid, and product yield at the end of the culture were measured. The results are shown in Table 1. Although most of the additives affected the maximum specific growth rate very little $\left(0.085-0.096 \mathrm{~h}^{-1}\right)$, adding oxaloacetic, fumaric, maleic and pyruvic acids to the medium reduced the growth rate. These results agree well with for those of Hijikata et al. (1). The $Y_{\mathrm{p} / \mathbf{x}}$ values for the two media containing citric acid or succinic acid was as high as 180 ; with the control medium it was 110 . Therefore we used a medium supplemented with each of them at a concentration of $2 \mathrm{~mm}$ in a continuous culture in a bubble column reactor. Air at an oxygen partial pressure of $0.21 \mathrm{~atm}$ was used in this experiment. The dilution rate was varied from 
Table 1. Effect of TCA cycle intermediates on growth and acetic acid production of Acetobacter aceti M23.

\begin{tabular}{|c|c|c|c|c|}
\hline \multirow{2}{*}{ Additive } & \multicolumn{4}{|c|}{ Parameters } \\
\hline & $\mu_{\mathrm{m}}^{a}, \mathrm{~h}^{-1}$ & $\Delta X^{b}, \mathrm{~g} / l$ & $\Delta P^{c}, \mathrm{~g} / l$ & $Y_{\mathrm{p} / \mathbf{x}}^{d}$ \\
\hline Control & 0.096 & 0.22 & 24.4 & 110 \\
\hline Citric acid & 0.092 & 0.26 & 47.5 & 180 \\
\hline Oxaloacetic acid & 0.058 & 0.18 & 19.8 & 113 \\
\hline Maleic acid & 0.077 & 0.32 & 39.8 & 103 \\
\hline Fumaric acid & 0.070 & 0.26 & 28.6 & 108 \\
\hline$\alpha$-Ketoglutaric acid & 0.092 & 0.33 & 50.8 & 153 \\
\hline Succinic acid & 0.085 & 0.31 & 56.0 & 181 \\
\hline Pyruvic acid & 0.068 & 0.30 & 35.2 & 136 \\
\hline
\end{tabular}

a Maximum specific growth rate.

$b$ Increase of cell concentration.

c Increase of acetic acid concentration.

d $\Delta P / \Delta X(\mathrm{~g} / \mathrm{g})$.

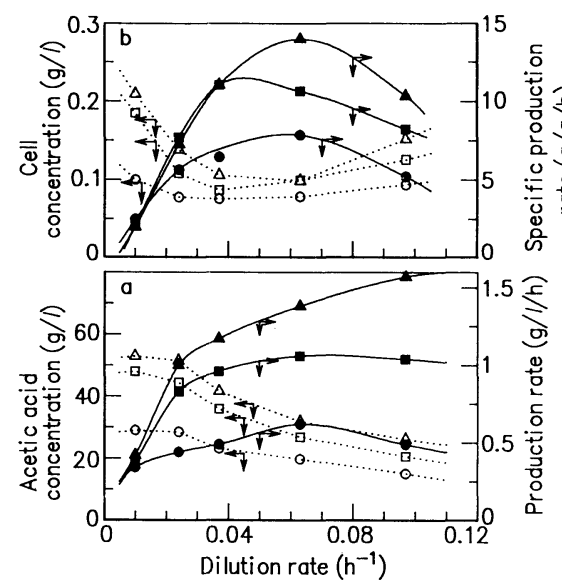

Fig. 1

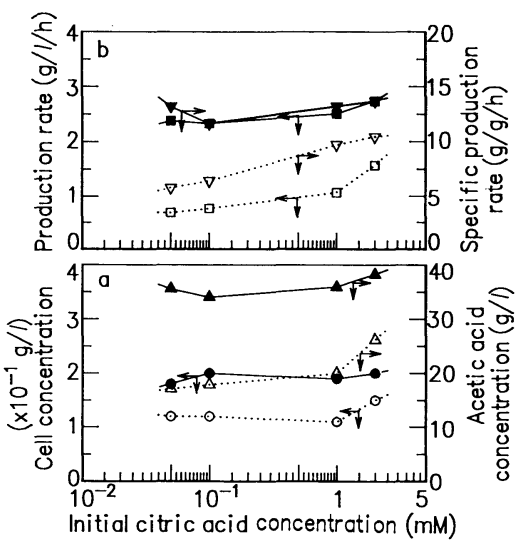

Fig. 2

Fig. 1. Acetic acid concentration and production rate (a), and cell concentration and specific acetic acid production rate (b) in continuous culture of $A$. aceti. The supplemented substance is: $\triangle \boldsymbol{\Lambda}$, citric acid; $\square \mathbf{\square}$, succinic acid; $\bigcirc \bullet$, none (control medium). Open symbols (dotted lines) and closed symbols (solid lines) in Fig. 1a denote acetic acid concentration $(\mathrm{g} / l)$ and production rate $(\mathrm{g} / \mathrm{l} / \mathrm{h})$, respectively. Open symbols (dotted lines) and closed symbols (solid lines) in Fig. $1 \mathrm{~b}$ denote dry cell concentration $(\mathrm{g} / l)$ and specific acetic acid production rate $(\mathrm{g} / \mathrm{g} / \mathrm{h})$.

Fig. 2. Concentrations of acetic acid and dry cells (a) and acetic acid production rate and specific production rate (b) with varying citric acid concentrations in the feed from 0.05 to $2 \mathrm{~mm}$ in the continuous culture at dilution rate of $0.097 \mathrm{~h}^{-1}$. Symbols in Fig. 2a: $\bigcirc \bullet$, dry cell concentration $(\mathrm{g} / l) ; \triangle \boldsymbol{\Delta}$, acetic acid concentration $(\mathrm{g} / l)$. In Fig. $2 \mathrm{~b}: \square \mathbf{\square}$, production rate $(\mathrm{g} / \mathrm{l} / \mathrm{h}) ; \nabla \boldsymbol{\nabla}$, specific acetic acid production rate $(\mathrm{g} / \mathrm{g} / \mathrm{h})$. Open and closed symbols denote parameters determined at oxygen partial pressure of 0.21 atm and 0.8 atm, respectively. 
$0.01 \mathrm{~h}^{-1}$ to $0.097 \mathrm{~h}^{-1}$. The results are shown in Fig. 1. The acetic acid concentration at a steady state clearly increased with the addition of any one of the additives. The specific production rate of acetic acid had a maximum value at the dilution rate of about $0.06 \mathrm{~h}^{-1}$, as was observed in a previous study(3). The cell concentrations increased slightly with the various dilution rates although the increase was rather small in comparison with the other culture parameters. The peak cell concentration also occurred previously at the dilution rate of $0.14 \mathrm{~h}^{-1}$ (washout rate was about $\left.0.17 \mathrm{~h}^{-1}\right)(5)$. The reason is left for further investigation. Citric acid enhanced the acetic acid production rate at the dilution rate of $0.097 \mathrm{~h}^{-1} 3.2$ times that of the control medium. Muller-Kraft and Babel(4) investigated the specific activity of TCA cycle enzymes in Acetobacter methanolicus, and reported that the specific activity of aconitase was the highest of all. Aconitase is the enzyme which catalyzes the reversible interconversion of citrate and isocitrate via the enzyme-bound intermediate cis-aconitate(2). The supplement of TCA cycle intermediates such as citric acid to the medium may favor the start up of TCA cycle reaction, especially at the beginning of the culture. However Hijikata et al. (1) reported that most effective metabolites were succinic acid and lactic acid in a modified Hromatka medium for acetic acid production in Acetobacter rancens. They found experimentally, using ${ }^{14} \mathrm{C}$-labeled metabolites, that parts of those effective metabolites were incorporated into cells.

Optimum citric acid concentration for acetic acid production

In an experiment with shake flask cultures we determined the optimum concentration of citric acid in medium for acetic acid production. The initial citric acid concentration was varied from 0 to $1 \mathrm{M}$ (Table 2). The maximum specific growth rate did not depend on the initial citric acid concentration. However the product yield coefficients $Y_{\mathrm{p} / \mathrm{s}}$ and $Y_{\mathrm{p} / \mathrm{x}}$ were maximal $(1.21(\mathrm{~g} / \mathrm{g})$ and $213(\mathrm{~g} / \mathrm{g}))$ respectively, at the intermediate citric acid concentration of $10 \mathrm{~mm}$. The estimated stoichiometrical value of $Y_{\mathrm{p} / \mathrm{s}}$ is $1.30 \mathrm{~g} / \mathrm{g}$ assuming $1 \mathrm{~mol}$ ethanol converted to $1 \mathrm{~mol}$ acetic acid. The $\mathrm{pH}$ of the medium decreased by $0.15,0.25,1.10$ and 2.01 with the addition of citric acid at concentrations of $5,10,100$ and $1,000 \mathrm{~mm}$, respectively. So the decrease in product yield at the high citric acid concentration may be caused by the unfavorable decrease of $\mathrm{pH}$.

Table 2. Effect of initial citric acid concentration on growth rate and product yield.

\begin{tabular}{cccccc}
\hline & \multicolumn{5}{c}{ Initial citric acid concentration $(\mathrm{mM})$} \\
\cline { 2 - 6 } Parameters & 0 & 1 & 10 & $1 \times 10^{2}$ & $1 \times 10^{3}$ \\
\hline$\mu_{\mathrm{m}}\left(\mathrm{h}^{-1}\right)$ & 0.063 & 0.053 & 0.066 & 0.058 & 0.060 \\
$Y_{\mathrm{p} / \mathrm{s}}(\mathrm{g} / \mathrm{g})$ & 0.87 & 1.12 & 1.21 & 1.11 & 1.06 \\
$Y_{\mathrm{p} / \mathbf{x}}(\mathrm{g} / \mathrm{g})$ & 134.6 & 181.8 & 213.0 & 187.7 & 168.0 \\
\hline
\end{tabular}


Effect of oxygen partial pressure on acetic acid production in a continuous culture using the medium containing citric acid

A continuous culture was conducted at a dilution rate of $0.097 \mathrm{~h}^{-1}$ to investigate the combined effect of oxygen partial pressure and citric acid concentration. The citric acid concentration in the feed medium was varied from $0.05 \mathrm{~mm}$ to $2 \mathrm{~mm}$ (Fig. 2). When the air with oxygen partial pressure of $0.21 \mathrm{~atm}$ was sparged in the bubble column reactor the concentrations of acetic acid and cells at a steady state were dependent on the feed citric acid concentration. The dissolved oxygen concentration was calculated as $0.15 \mathrm{ppm}$ from the acetic acid production rate of $0.69 \mathrm{~g} / \mathrm{l} / \mathrm{h}$ in the medium containing $0.05 \mathrm{~mm}$ citric acid, assuming a saturated dissolved oxygen concentration of $7.6 \mathrm{ppm}$ and volumetric oxygen transfer coefficient of $50 \mathrm{~h}^{-1}$. The dissolved oxygen concentrations in the culture was almost depleted throughout these experiments. However when the oxygen-enriched air (oxygen partial pressure, $0.8 \mathrm{~atm}$ ) was used, the concentrations of the acetic acid and cells and the acetic acid production rate were virtually constant independent of the feed citric acid concentration. This indicates that the acetic acid production depended on citric acid concentration only under conditions of insufficient oxygen supply, such as an oxygen partial pressure of 0.21 atm. In $A$. aceti cells the dissolved oxygen is used competitively both for the ethanol oxidation to the electron transfer system in the acetic acid production and for the metabolism of glucose to the electron transfer system via the TCA cycle. But since most of dissolved oxygen is consumed for the ethanol oxidation, the rate of metabolism of glucose to the TCA cycle decreases concomitantly. Therefore the TCA cycle intermediates necessary for the cell growth are depleted. This in turn reduces the cell concentration and the acetic acid production rate. The TCA cycle intermediates added to the basal medium may be used to stimulate the metabolic cycle for cell growth, even when there is an insufficient oxygen supply, and thus keep the acetic acid production rate from deteriorating. The compensatory effect of the supplemented TCA cycle intermediates on acetic acid production must be important especially for the acetic acid production in a high cell-density culture (6), since dissolved oxygen in the culture tends to become depleted. It is expected that the effect is also significant when the bioreactor for acetic acid production is scaled up for industrial purposes. In a large-scale fermenter local regions of dissolved oxygen depletion sometimes develop even though the average dissolved oxygen concentration in the fermenter is kept higher than a critical value.

\section{REFERENCES}

1) Hijikata, Y., Okumura, H., and Terui, G., Studies on factors to promote acetic acid fermentation by Acetobacter rancens (II) Effect of some simple metabolites. J. Ferment. Technol., 50: 7-12 (1972).

2) Lehninger, A. L., Biochemistry: The tricarboxylic acid cycle and the phosphogluconate pathway, Worth Publishers, Inc., New York (1975), p. 443-476.

3) Mori, A. and Terui, G., Kinetic studies on submerged acetic acid fermentation II. Process kinetics. J. Ferment. Technol., 50: 70-78 (1971). 
4) Muller-Kraft, G. and Babel, W., The tricarboxylic acid cycle in Acetobacter methanolicus. J. Basic Microbiol., 28: 95-99 (1988).

5) Park, Y. S., Ohtake, H., Fukaya, M., Okumura, H., Kawamura, Y., and Toda, K., Acetic acid production using a fermenter equipped with a hollow fiber filter module. Biotechnol. Bioeng., 33: 918-923 (1989).

6) Park, Y. S., Ohtake, H., Fukaya, M., Okumura, H., Kawamura, Y., and Toda, K., Enhancement of acetic acid production in a high cell-density culture of Acetobacter aceti. J. Ferment. Bioeng., 68: 315-319 (1989).

7) Samson, F. E., Katz, A. M., and Harris, D. L., Effects of acetate and other short-chain fatty acids on yeast metabolism. Arch. Biochem. Biophys., 54: 406-423 (1955). 